This full text version, available on TeesRep, is the PDF (final version) of:

Ohlander, A. et. al. (2010) 'Assembly of a polymer lab-on-chip device for impedimetric measurements of D-dimers in whole blood', 60th electronic components \& technology conference, Las Vegas, Nevada, June 1-4, in 2010 proceedings. IEEE, pp.1004-1009.

For details regarding the final published version please click on the following DOI link: http://dx.doi.org/10.1109/ECTC.2010.5490824

When citing this source, please use the final published version as above.

Copyright (C) 2005 IEEE. This material is posted here with permission of the IEEE. Such permission of the IEEE does not in any way imply IEEE endorsement of any of Teesside University's products or services. Internal or personal use of this material is permitted. However, permission to reprint/republish this material for advertising or promotional purposes or for creating new collective works for resale or redistribution must be obtained from the IEEE by writing to pubs-permissions@ieee.org.

By choosing to view this document, you agree to all provisions of the copyright laws protecting it.

This document was downloaded from http://tees.openrepository.com/tees/handle/10149/114365 Please do not use this version for citation purposes.

All items in TeesRep are protected by copyright, with all rights reserved, unless otherwise indicated. 


\title{
Assembly of a Polymer Lab-on-Chip Device for Impedimetric Measurements of D-Dimers in Whole Blood
}

\author{
A. Ohlander ${ }^{1}$, C. Strohhöfer ${ }^{1}$, K. Bock ${ }^{2,1}$ S. M. Scott ${ }^{3}$, Z. Ali ${ }^{3}$, Peter Musil ${ }^{4}$, Jan Kyselovic ${ }^{4}$ \\ ${ }^{1}$ Fraunhofer Institute for Reliability and Microintegration, Hansastrasse 27d, 80686 Munich, Germany, \\ ${ }^{2}$ Technical University of Berlin, Strasse des 17 Juni 135, 10623 Berlin, Germany \\ ${ }^{3}$ Teesside University, School of engineering and science, Middlesbrough, Tees Valley, TS1 3BA, UK \\ ${ }^{4}$ Pharmaceutical Faculty Comenius University, Kalinciakova 8, Bratislava, 832 32, Slovak Republic
}

\begin{abstract}
This paper reports the development and characterisation of an assembly technology for a polymer lab-on-chip. The system consists of a $150 \mu \mathrm{m}$ deep hot embossed microfluidic channel in polycarbonate and $\mathrm{Au}$ electrodes fabricated separately by photolithography on polyethylenenaphthalate. The system is designed for impedimetric immunoassay detection in whole blood. Electrode layer and microfluidic substrate are joined by means of a $50 \mu \mathrm{m}$ thick double-sided medical grade adhesive tape, adjusted with an optical alignment system. The bond proved to be liquid tight at room temperature. An alignment accuracy of $34 \mu \mathrm{m}(+/-19 \mu \mathrm{m})$ evaluated over a set of 23 samples, was achieved. The effect of alignment accuracy of the intermediate adhesive film on whole blood flow properties in the device is studied. Already an alignment error of $70 \mu \mathrm{m}$ increases the flushing out time of whole blood by approximately $20 \%$.
\end{abstract}

\section{Introduction}

The increasing interest in point-of-care devices and labon-chips to reduce cost of diagnosis and increase the well being of patients calls for low cost, high throughput manufacturing technologies to realise the commercialisation of such systems. For this reason, the use of polymers and thermoplasts to produce micro systems for diagnostic purposes has increased due to their attractive features such as low material cost, diversity in chemical and physical functionality and their diverse processability [1].

A critical step in the manufacturing chain of a lab-on-chip, and often seen as a bottle neck in the production, is the joining and sealing of microfluidic systems [2]. General hurdles to overcome are bonding of different materials onto each other, maintaining for example biological and fluidic functionality during the bonding process and adjusting microscale elements onto each other with high precision. One popular method for sealing of thermoplastic fluidics is by sealing the channel with a lid of the same material by fusion bonding (thermal or solvent) [3]. For the joining of layers of different materials, which allow for greater flexibility of functionality and processing, the introduction of an intermediate adhesive layer is seen as a low-cost alternative [2]. Further, polymeric adhesives can among others offer moisture and chemical resistance, thermal and electrical insulation and are at hand in a vast variety of chemical compositions compatible with various materials [4]. Lamination of such intermediate adhesive layers is a popular sealing technology as commercial lamination machines are at hand and a manifold of lamination films are commercially available [3]. From the precision point of view, lamination films also has advantages in handling compared to liquid adhesives as the application of a liquid adhesive might require extensive optimisation work in order not to clog any channels or block structures [5]. Using laminate foils to seal fluidics or to build up multilayer structures was demonstrated by several authors [6],[7], [8]. Alignment of layers and components can for example be performed by integrating pin and hole structures on the layers to be aligned [8], [9], applying a registration frame [10] or using standard silicon wafer bonding equipment [11]. In the latter, alignment accuracy on the micrometer scale for silicon systems was reported. High accuracy alignment of polymer systems is however facing additional challenges beyond those of silicon systems. Dimensional instabilities deriving from the responsive nature of polymers to changes in temperature and chemical environment during processing complicates registration.

In this article, a double-sided adhesive foil lamination process for the joining of two individually manufactured functional polymer layers is reported. Alignment was performed in a semi-automated process using an optical alignment system for silicon chip bonding, adapted to handle the larger micro fluidic substrate. The system was designed for impedimetric detection of the thrombosis marker D-dimer in whole blood. Special attention was given to the thermal and chemical assembly conditions, with the aim to provide a room temperature and chemically inert environment throughout the whole assembly. We aimed at providing an environment during assembly, in which the biomolecules of the sensor do not lose their functionality. Further, we demonstrate the impact of misalignment on whole blood flow in the microfluidics. Misalignment can here affect the overall performance of the microsystem with respect to analysis times and measurement accuracy.

\section{System specification}

The system consists of two functional parts: first, a hot embossed $150 \mu \mathrm{m}$ deep and $500 \mu \mathrm{m}$ wide channel structure in polycarbonate with three chambers with a width of $2 \mathrm{~mm}$, and second, gold electrodes fabricated by photolithography on polyethylenenaphthalate (PEN). The choice of different substrate materials for the two components was dictated by processing conditions for materials, biomolecule immobilisation and bioassay. The electrode layer contains electrodes for the impedimetric measurement as well as control electrodes for localization of the fluids. The electrode layer and microfluidic channel, was joined with a medical grade, pressure sensitive double-sided adhesive foil (Fig 1). In order for the electrodes to be in contact with the analytic fluid, the adhesive foil is structured in the shape of the 
microfluidic channel. Using a pressure sensitive adhesive foil avoids the necessity of a curing step in the bonding process that may damage the sensing biomolecules immobilized on the impedimetric electrodes. Curing of adhesives often involves UV- light or high temperatures and out gassing of resins which are disturbing the environment for the biomolecules causing them to lose their functionality.

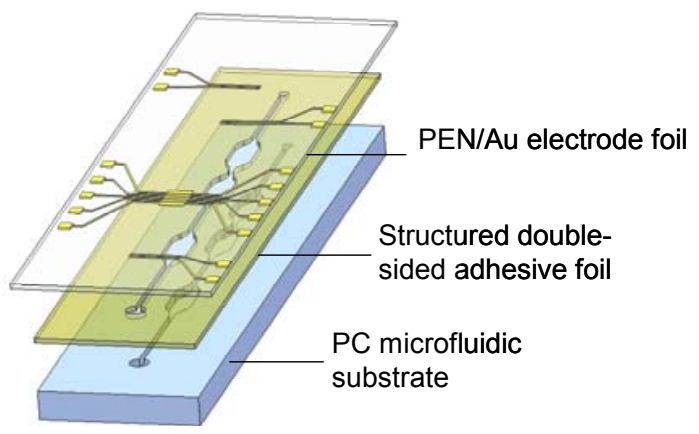

Figure 1. Schematic of the components and their assembly into a lab-on-chip.

The impedimetric measurement of the D-dimer interaction with the specific antibodies on the electrodes needs to be performed in buffer solution. This means that after a reference measurement in buffer solution, the whole blood sample is transported into the analysis chamber for incubation and then transported out of the chamber again to be able to perform the actual measurement. With the risk of blood cells adhering on to the electrodes and hence affecting the impedance, no whole blood remnants may be present in the analysis chamber during the measurement. Misalignment of the adhesive foil relative to the microfluidic cartridge causes dead spaces and cavities and result in distorted liquid flow in the system. The flushing of the analysis chamber is consequently aggravated, resulting in increased analysis times. Therefore, optimal functionality of the system requires accurate alignment of the three layers in fig. 1 with respect to each other both in vertical and horizontal direction. The alignment needs to be sufficiently accurate over the whole length of the lab-on-achip, i.e. several centimeters.

\section{Experimental}

The microfluidic channels were manufactured from 3.0 $\mathrm{mm}$ thick polycarbonate sheets by hot embossing.

Gold electrodes were photolithographically manufactured on $125 \mu \mathrm{m}$ thick polyethylenenaphtalate (PEN) sheets. The 20 x $70 \mathrm{~mm}^{2}$ large electrode layers were cut from the sheets by frequency-tripled $\mathrm{Nd}: \mathrm{YVO}_{4}$-laser with an accuracy of below $20 \mu \mathrm{m}$.

The $50 \mu \mathrm{m}$ thick double sided adhesive tape (AR care 8890, Adhesive Research Ireland Ltd.) was cut by Nd:YVO ${ }_{4}^{-}$ laser with the outer contour of the fluidic cartridge and the inner contour of the microfluidic channel.

The electrode layer and the microfluidic body were joined in a two-step lamination procedure. The foil was adjusted in between the two layers by using an optical alignment system for silicon chip bonding, adapted to handle larger micro fluidic substrates. Blister-free, full adhesive contact onto the cartridge was assured by means of pressure exerted by a metal roll onto the adhesive foil and cartridge after each bonding process. Bonding was performed at room temperature.

The alignment accuracy of the assembly approach was evaluated by taking microscopic images at specific locations on the assembled system and comparing the measured alignment with the design.

The whole blood flow in the devices was studied by deliberately fabricating devices with mismatches between the adhesive foil and the micro fluidic channel with 20,70, 90, 120 and $150 \mu \mathrm{m}$. Fluidic systems without electrodes on the PEN foil were used for this experiment. Chips were mounted under a microscope and filled to the inlet with $10 \mathrm{mM}$ PBS (Phosphate Buffered Saline). A drop of heparinised human whole blood was applied onto the inlet and sucked into the system. The blood sample was held still for two minutes to simulate an incubation step and then pushed back towards the inlet reservoir with a speed of $0,45 \mu 1 / \mathrm{s}$. The flushing behaviour of the five micro fluidic systems was monitored by video microscope. Pictures were extracted from the video and the intensity over the channel was analysed using ImageJ image analysis software

\section{Bonding}

Using the two step lamination process, the fluidic channel and the electrode sheet were joined to produce a closed system. A picture of the bonded microfluidic chip with integrated impedimetric sensors can be seen in figure 2 .

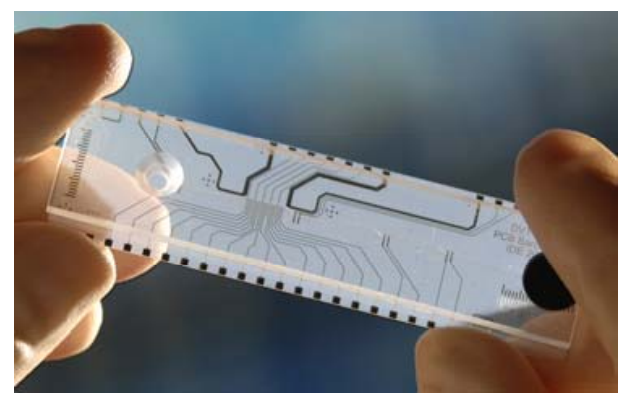

Figure 2. An assembled microfluidic cartridge with gold electrodes for impedimetric measurements.

The system proved to be liquid tight and exhibited a bonding shear strength of $0,9 \mathrm{MPa}$ measured according to DIN EN 2243-1. This is easily sufficient for microfluidic applications.

\section{Alignment}

The components to be assembled for the present microsystem are in the size of $20 \times 70 \mathrm{~mm}^{2}$. Due to the channel and electrode geometry, accurate alignment is required over at least a distance of $55 \mathrm{~mm}$ (the length of the microfluidic channel), far surpassing the requirements on Sichip bonding. This emphasises the need for a highly accurate assembly process. The layout of the electrode layer containing five interdigitated impedance electrodes and four resistive elements for fluid localization can be seen in figure 3 . The 
five impedance electrodes are denoted $\mathrm{C} 1-\mathrm{C} 5$ and the fluid control electrodes R1-R4

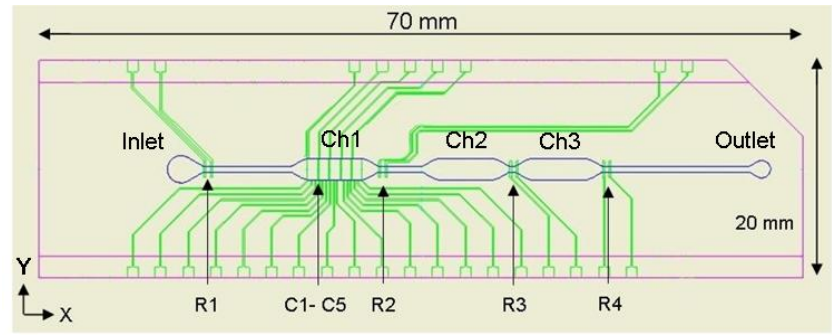

Figure 3. Layout of fluidic channel (blue) and measurement electrodes (green). C1-C5 denotes the impedimetric electrodes and R1-R4 the fluid control electrodes.

The accuracy of alignment using the optical alignment system was determined by comparing positions of electrodes with respect to the fluidic channel. Figure 4 illustrates exemplarily the features used for determining the horizontal alignment accuracy (x-direction) at electrode R3. a)

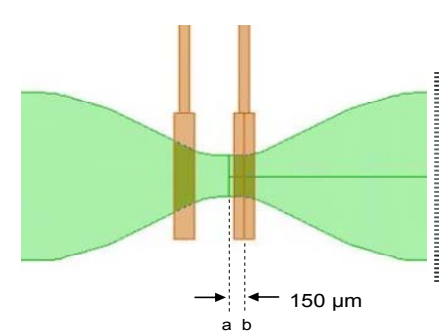

b)

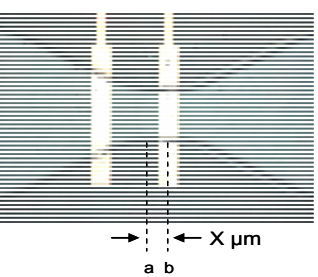

Figure 4. a) In the design, the centre of conductor b on electrode R3 and the centre between the two fluidic chambers are used as a reference. The geometry in (a) is compared with the same geometry in the device (b).

One major challenge in polymer processing is control of dimensional stability as the nature of polymers renders them susceptible to chemical and thermal changes in the environment. For example, lithographic processing and screen printing of layers on a $50 \mu \mathrm{m}$ thick PET foil were reported to cause $0,25 \%$ shrinkage of the substrate during processing [12]. In hot embossing, the degree of shrinkage and warpage can directly be derived from the thermomechanical history of the moulded piece [13]. Consequently, optimization and control of process parameters is of utmost importance to achieve features according to design in the fabrication of polymer microparts. Dimensional variance of polymer microstructure complicates alignment and may cause mismatch of layers relative to each other and hence reduce the alignment accuracy. As can be seen in figure 4b, a mismatch between the microfluidic channel and the adhesive foil is present. There is no mismatch between the electrode layer and the adhesive foil. It is assumed that thermal shrinkage of the polycarbonate during the hot embossing is causing the mismatch and will have to be adjusted by proper moulding conditions. The assembly method was therefore evaluated by comparing the electrodes position relative the adhesive foil.

Similar measurements on the electrodes C1-5 and R2-4 were made. By this method, the alignment accuracy of the electrode foil relative to the microfluidic cartridge could be determined over a length of $25 \mathrm{~mm}$ on the cartridge. The average misalignments in vertical (Y) and horizontal (X) direction on a set of 10 cartridges are represented in table 1.

\begin{tabular}{|c|c|}
\hline \multicolumn{2}{|c|}{$\begin{array}{c}\text { Table 1. Average alignment accuracy on a set of } 10 \\
\text { samples at four locations }\end{array}$} \\
\hline Electrode & Alignment accuracy \\
\hline C1-C5 & $30.76(+/-14.26)$ \\
\hline R2 & $13.74(+/-8.36)$ \\
\hline R3 & $14.26(+/-10.74)$ \\
\hline R4 & $28.71(+/-14.26)$ \\
\hline R3 (horizontal) & $25.70(+/-16.29)$ \\
\hline
\end{tabular}

Due to the lack of sharp edges and fix points on the microfluidic structure, the horizontal alignment evaluation could only be performed on electrode R3. This electrode is placed between two chambers making it possible to find its position relative to the centre line between the two chambers. The data shows that the assembly approach can deliver an alignment accuracy of 31 microns in lateral direction and 26 in horizontal. Another 13 cartridges were assembled, this time by a different operator to get operator independent results. The distribution of the alignment accuracy of all assembled cartridges is represented in figure 5 .

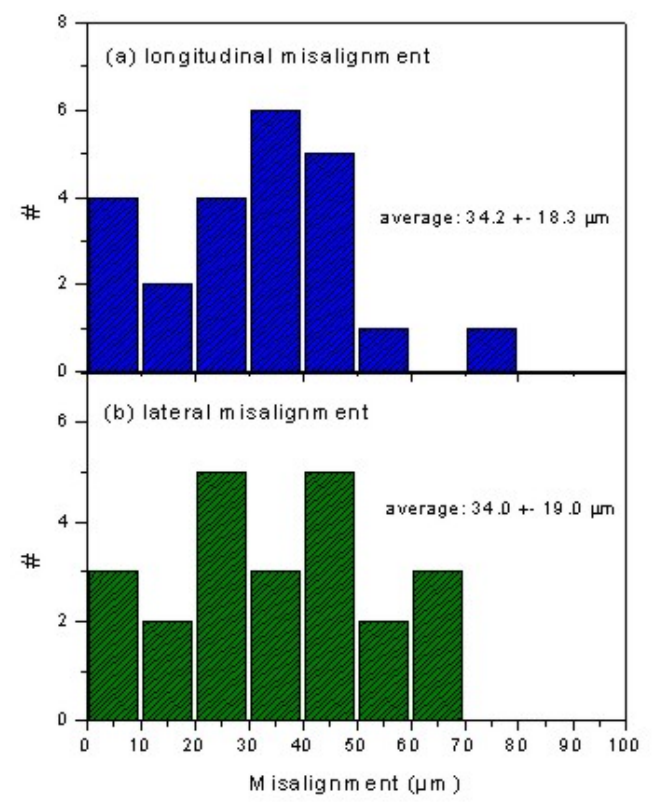

Figure 5. Distribution of lateral and horizontal misalignment on the set of 23 cartridges.

The lateral misalignment average over all samples amounts to $34.0 \pm 19.0 \mu \mathrm{m}$ and in longitudinal direction to 
$34.2 \pm 18.3 \mu \mathrm{m}$. The distribution shows clearly that in both directions, the accuracy is centered around the average value. The change of operator produces alignment results comparable to the first 10 cartridges assembled.

Due to the wide range of polymer materials and polymer processing technologies resulting in micro structures with a variety of texture and characteristics, it is in general difficult to compare different alignment methods for polymer microsystems with each other. Few examples are to be found in the literature where alignment accuracy is reported on a statistical base and there seem to be a variance in how the term alignment accuracy is interpreted and measured. Weigl et al, reported a width and depth tolerance of 1 and $10 \mu \mathrm{m}$ respectively for channels of 100-3 $000 \mu \mathrm{m}$ in width and 50$400 \mu \mathrm{m}$ in depth for systems of 4-12 lamination layers aligned by using a registration frame [10]. In this case, the layers were, however cut by laser and consequently not exposed to any thermal stress during processing, minimizing dimensional instability. Furthermore, all 4-12 layers were manufactured by the same process increasing dimensional homogeneity among layers and leading to a high alignment accuracy. In this article, an assembly technology for three layers manufactured by three distinctly different technologies is presented. A more comparable example in the literature can be found in the work of $\mathrm{Fu}$ et al, where an alignment tolerance of $+/-25 \mu \mathrm{m}$ in a system consisting of three hot embossed polysulphone layers, three structured metal layers and five adhesive layers structured by punch technology is reported [8]. The alignment was performed by pin and hole structures on the different layers. It is however not revealed how the tolerance was determined and on which statistical base.

\section{Alignment and fluidic properties}

One main functionality in lab-on-chips is the transport of fluidic samples to different locations on the chip. Consequently, high accuracy alignment of the layers in the system is needed in order not to distort channel geometries and hence not to disturb the flow properties. Our alignment technology provides us with the means to study the effect of misalignment on the fluidic properties of the system. We here exemplarily present the effect of a misaligned adhesive foil. In order to study this effect in the system, cartridges with controlled misalignments of the adhesive foil relative to the microluidic channel were fabricated. The cross section of a channel with misaligned intermediate adhesive foil is schematically shown in figure 6.

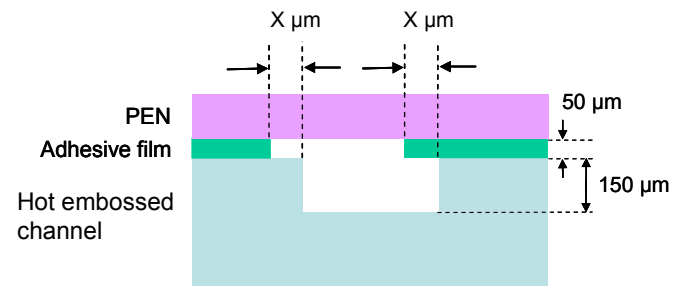

Figure 6. Schematic of microfluidic system with variable misalignment $\mathrm{X} \mu \mathrm{m}$ of adhesive foil relative to channel.
This adds a $50 \mu \mathrm{m}$ high and $\mathrm{x} \mu \mathrm{m}$ wide recess to the normally $200 \mu \mathrm{m}$ deep and $500 \mu \mathrm{m}$ wide channel. The accuracy of the misalignments reported here is determined by the $20 \mu \mathrm{m}$ wide edge contour of the adhesive foil, originating from laser cutting. Five cartridges with misalignments of 20, $70,90,120$ and $150 \mu \mathrm{m}$ were assembled. The cartridges were filled with heparinized human whole blood up to the outlet on the second blood reservoir chamber ( $\mathrm{Ch} 3$ in figure 3). An incubation step of two minutes was simulated and subsequently the blood was flushed out with buffer solution towards the inlet. The flushing was monitored by video microscope. We aim to determine the influence of the recess's width $\mathrm{x}$ on the flushing behavior of the chamber containing the interdigitated electrodes as this is the site for the impedimetric measurement (Ch1, fig 3). For this purpose, snapshots were extracted from the videos at different points in time. An example of snapshots and corresponding intensity profile can be seen in figure $7 \mathrm{a}$ and $\mathrm{b}$ respectively.

a)

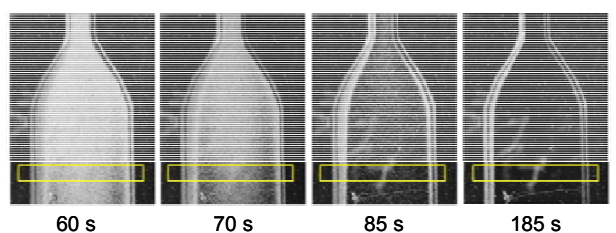

b)

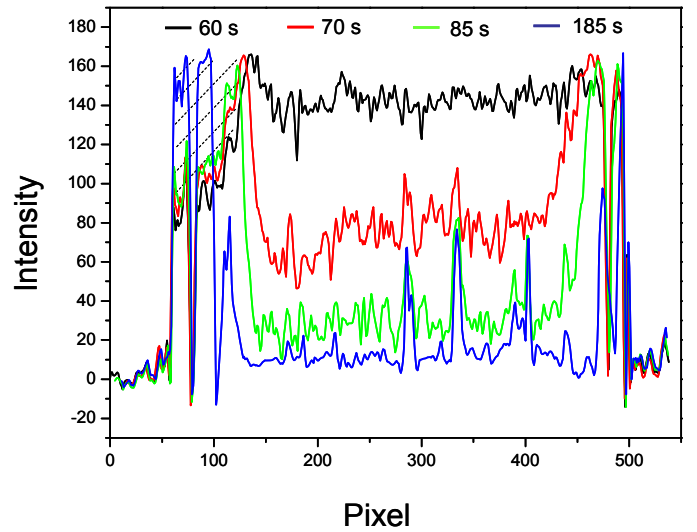

Figure 7. (a) Snapshots taken at 60, 70, 85 and 185 seconds after initiating the flushing of the chamber. The cartridge has a $70 \mu \mathrm{m}$ misalignment of the adhesive foil relative the microfluidic channel. (b) The corresponding intensity profiles of the yellow box of the four snapshots in a)

In figure 7a, four gray scale microscope images of a cartridge with $70 \mu \mathrm{m}$ misalignment between adhesive foil and microfluidic channel at four different times during the flushing process are seen. The misalignment between adhesive foil and microfluidic is visible as a double contour along the fluidic structure. On the left side of the images is the recess and on the right side the adhesive foil overlapping into the channel. This far in the flushing process, the blood has been diluted with buffer to the extent that single blood cells can be seen as white dots, originating from light scattering by the individual cells. The videos were shot in darkfield mode, 
which enhances contrast in transparent samples, making both blood cells and the polycarbonate cartridge clearly visible. Dark field mode is generally used when observing biological samples as it avoids heating of the sample by the microscope light beam, since the sample only is exposed to scattered light from the light source. The blood is gradually flushed out of the chamber over the sequence of snapshots seen in figure $7 \mathrm{a}$. It can be seen how the $70 \mu \mathrm{m}$ wide cavity on the left side of the channel caused by the misaligned adhesive foil, affects the flow in the chamber so that more blood cells remain close to the left chamber wall than to the right one. The impact of the adhesive foil of the right chamber side on the flow is apparently less severe. To quantify the amount of blood cells remaining in the chamber, intensity profiles across the channel were calculated at the fixed location indicated by boxes, in the images in figure 7a. The boxed areas were integrated in channel direction to reduce statistical and background errors. In figure $7 \mathrm{~b}$, the intensity plots of the marked areas are shown. Our signal is caused by light scattering from the blood cells. The more cells present in the channel, the higher the intensity of the scattered light. An empty channel causes a low intensity signal close to zero, the remainder being background signal from inhomogeneities in the polycarbonate substrate and PEN foil. However, if the density of cells is too high, absorption by the blood cells dominates scattering and reduces the image intensity. This means that care needs to be taken when interpreting the intensity plots. An example of this effect can be discerned in figure $7 \mathrm{~b}$. On the first three measurements $(60,70$ and 85 seconds), the curves decline to an intensity value of sixty on the left side of the graph. This is lower than the peaks achieved in the same area for the curve at $185 \mathrm{~s}$ in the same region. In reality, there are more blood cells present on the left side of the chamber in the images at 60,70 and 85 than at $185 \mathrm{~s}$, so the intensity profiles do not entirely reflect the amount of blood cells present in the chamber. To estimate a value of this error, the area shaded in the figure was integrated and multiplied by two, assuming that the intensity value cannot exceed double the value of the decrease. This results in an estimated error of $5 \%$ relative the start blood volume at $60 \mathrm{~s}$ after flushing. This error decreases as the blood cell density decreases to become zero for the last 100 seconds of the recorded flushing behavior.

Due to these measurement uncertainties, it is difficult to distinguish the flow properties in the cartridges with misalignment differences of only $20 \mu \mathrm{m}$. For this reason, only the flush behavior of the 20,70 and $150 \mu \mathrm{m}$ misaligned samples was further evaluated. The integrals of the intensity profiles were calculated and plotted. Figure 8 shows the normalized intensity integrals across the microfluidic chambers at different times during the flushing. The first intensity integral was determined after 60 seconds of flushing in the system.

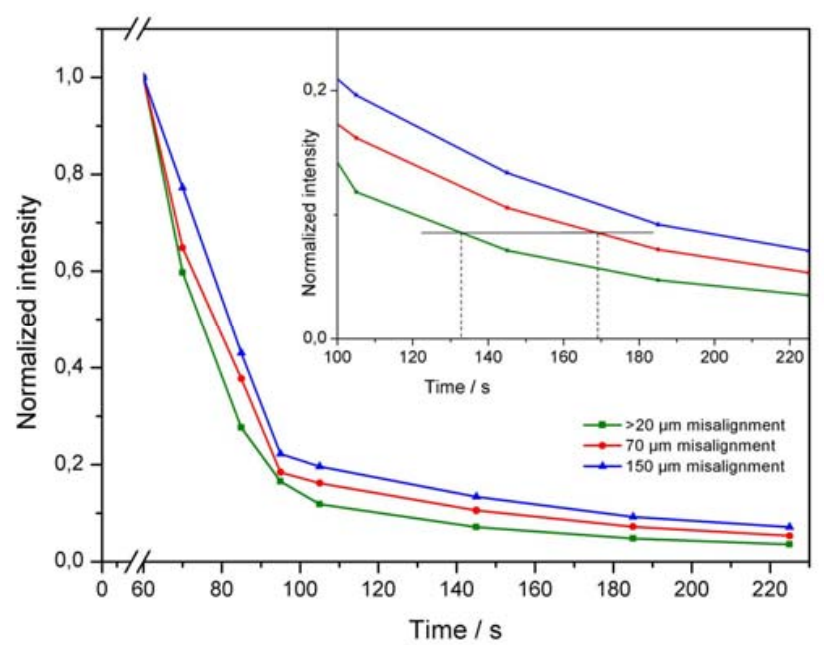

Figure 8. Intensity of light scattered from blood cells, measured in cartridges with misalignments of 20,70 and 150 $\mu \mathrm{m}$ relative flush time. The light intensity serves as a measure for the contamination of the channel with blood cells.

Overall, it can be seen that within the first 30 seconds of the flushing curves plotted, about $70-80 \%$ of the blood cells are removed from the chamber in the three cases. Subsequently, it takes about 130 seconds for the average blood cell density to decrease from about 20 to only $5 \%$ of the start amount of blood in the channel. Apparently, most of the flush time is spent on removing the last blood cells, that remain in the corners of the square cross section of the fluidic channel, out of the system. This is true even for the cartridge with $<20 \mu \mathrm{m}$ misalignment, which serves here as reference for perfect alignment. The inset of figure 8 compares the flush time needed to reach a level of scattered intensity in the misaligned cartridges of about $5 \%$ of their initial value when starting the measurement. With the help of a horizontal line crossing the curves in the inset in figure 8 , one can read out from the graph that a misalignment of $70 \mu \mathrm{m}$ of the adhesive foil relative to the microfluidic channel already results in an increased flush time of about $20 \%$. The larger the misalignment, the higher is the number of blood cells remaining in the channel at a given time. It can also be seen that in all three cases, still after almost four minutes flush time there are still some blood cells left in the chamber. This value increases to $7 \%$ for a $150 \mu \mathrm{m}$ misalignment compared to only $3 \%$ for a $<20 \mu \mathrm{m}$ misaligned adhesive foil. These results indicate that accurate alignment of layers onto each other in a microfluidic system is of utmost importance to maintain channel dimensions and also to reduce analysis times. They also emphasise the need for control of dimensional stability during processing of multilayer systems.

\section{Conclusions}

In this article we described a robust, high accuracy, room temperature assembly technology for polymer microfluidic devices. Using an intermediate laminate bonding film makes it possible to create a hybrid material system from two materials incompatible for thermal or solvent bonding, but 
independently possessing qualities that are highly desirable for manufacturing and performance. A liquid tight, blister free bond between the microfluidic and the electrode layer was created. With our assembly technology we have shown that highly accurate alignment of intermediate bonding layers is crucial to maintain desired channel dimensions and hence to reduce analysis times in lab-on-chips can be achieved. The system and assembly approach will be tested for its bio assaycompatibility in our lab-on-chip processing unit.

\section{Acknowledgments}

This work was supported in part by the European Union under the Marie Curie program MAPTech (Contract MESTCT-2005-020310) and the specific targeted research project DVT-IMP (contract \# INFSO-IST-034256). We thank Mathias Kuphal for providing us with the $\mathrm{Au}$ on PEN electrode layers and Dieter Bollman for laser cutting of the adhesive foil.

\section{References}

[1] Heckele M. et al, "Review on micro molding of thermoplastic polymers", J. Michromech. Michroeng., 14(2004) R1-R14.

[2] Velten T. et al, "Packaging of Mio-MEMS: Strategies, Technologies and applications" IEEE Transaction on Advanced Packaging, Vol. 28, No. 4, November 2005.

[3] Tsao C-W. et al, "Bonding of thermoplastic polymer microfluidics", Microfluid Nanofluid (2009) 6:1-16.

[4] Baldan A., "Review: Adhesively-bonded joints and repairs in metallic alloys, polymers and composite materials: adhesives, adhesion theories and surface pretreatment", Journal of Material Science 39 (2004) 149.

[5] Han J. et al, "UV adhesive bonding technique in room temperature for plastic lab-on-a-chips", 7th International Conference of Miniaturized Chemical and Biochemical Analysis Systems, October 5-9, 2003, Squaw Valley, California, USA.

[6] Huang F-C. et al, "CE chips fabricated by injection molding and polyethylene/thermoplastic elastomer film packaging methods", Electrophoresis 2007, 28, 11301137.

[7] Paul D. et al, "Lamination-based rapid prototyping of microfluidic devices using flexible thermoplastic substrates", Electrophoresis 2007, 28, 1115-1122.

[8] $\mathrm{Fu} \mathrm{C}$. et al, "Magnetically driven micro ball valves fabricated by multilayer adhesive film bonding", $J$. Micromech. Microeng. 13 (2003) S96-S102.

[9] Mossa E. D et al, "A fabrication technology for multilayer polymer-based microsystems with integrated fluidic and electrical functionality", Sensors and Actuators B xxx (2006) $x x x-x x x$.

[10] Weigl B. H. et al, "Design and rapid prototyping of thinfilm laminate-based microfluidic devices", Biomedical Microdevices 3:4, 267-274, 2001.

[11] Niklaus F. et al, "A method to maintain wafer alignment precision during adhesive wafer bonding", Sensors and Actuators A 107 (2003) 273-278.
[12] Strohhöfer C. et al, "Roll-to-roll microfabrication of polymer microsystems", Measurement + Control, Vol 40/3 april 2007.

[13]Worgull M. et al, „Analyse des Mikroheißprägeverfahrens“, Dissertation, ISSN 0947-8620, Forschungs Zentrum Karlsruhe GmbH, Germany. 